\title{
Small gain theorem for distributed feedback control of Sturm-Liouville dynamics
}

\author{
Boe-Shong Hong \\ Department of Mechanical Engineering, National Chung Cheng University, Chia-Yi 62012, Taiwan
}

Email address:

imehbs@ccu.edu.tw

\section{To cite this article:}

Boe-Shong Hong. Small Gain Theorem for Distributed Feedback Control of Sturm-Liouville Dynamics. Applied and Computational Mathematics. Vol. 3, No. 5, 2014, pp. 217-224. doi: 10.11648/j.acm.20140305.14

\begin{abstract}
This paper constructs the small-gain theorem upon a general class of Sturm-Liouville systems. It appears that the feedback connection of two Sturm-Liouville sub-systems is guaranteed of well-posedness, Hurwitz, dissipativity and passivity in $L_{2}$-spaces provided the loop gain is less than 1. To construct the theorem, spatiotemporal transfer-function and geometrical isomorphism between the space-time domain and the mode-frequency domain are developed, whereof the $H_{\infty}$-norm is extended to be $2 \mathrm{D}-H_{\infty}$ norm in mode-frequency domain. On grounds of this small-gain theorem, robust performance of any Sturm-Liouville plant can be formulated as robust stability of a feedback connection, whereupon feedback syntheses can be performed via modal-spectral $\mu$-loopshaping.
\end{abstract}

Keywords: Small Gain Theorem, Distributed Control, Robust Control, nD Transfer Function

\section{Introduction}

The small-gain theorem says that the feedback connection of two systems is guaranteed of dissipativity in $L_{2}$-spaces provided the product of the system gains is less than 1 . Through Small Gain theorem (SGT), robust performance of any generalized plant that includes performance requirements and modelling uncertainties can be transformed into robust stability of a feedback-connection of nominal plant and uncertainty. Thereupon feedback syntheses are performed via frequency-domain loopshaping or state-space bounded-real inequalities, which is beyond the reach of Circle or Popov criterion. Therefore, SGT is usually regarded as the first law as to Robust Control.

Consider a feedback loop $G$ interconnected by two systems: $M$ and $\Delta$ that are minimum dynamics to realize their input-output operations. Dependent on what types of systems $M$ and $\Delta$ are considered, there are four classes of SGT organized as follows:

(C1) In $L_{2}$-spaces, if the product of system gains $\|M\|\|\Delta\|<1$, then the feedback loop $G$ is guaranteed of dissipativity for generic dynamics $M$ and $\Delta$ [1]. With the assumption that the feedback-loop is well-posed, $G$ is further guaranteed of Lyapunov passivity.

(C2) In Banach spaces, the loop gain $\|M \Delta\|<1$ guarantees the feedback loop $G$ to be well-posed and dissipative, as both $M$ and $\Delta$ are of linearity and infinite states. This can be derived from elementary functional analysis, say [2].

(C3) As both $M$ and $\Delta$ are of linearity and finite states, the loop gain $\|M \Delta\|<1$ in $L_{2}$-spaces guarantees the feedback loop $G$ to be well-posed, dissipative, passive and Hurwitz. This has been largely documented in control literature, say in [3-4].

(C4) As $M$ and $\Delta$ are Sturm-Liouville dynamics in properly chosen spatiotemporal $L_{2}$-spaces, the loop gain $\|M \Delta\|<1$ guarantees the feedback loop $G$ to be well-posed, dissipative, passive and Hurwitz in some sense to be defined. This paper is aimed to explore this class of SGT.

Civilization has been encountered a great quantity of Sturm-Liouville plants- heat transfers, acoustic waves, structure vibrations, quantum mechanics, electromagnetic waves, non-Fourier heat transfers [5-6], thermoacoustic vibrations [7], and so on. In a bounded region, Sturm-Liouville dynamics mostly behave as standing waves, wherein modes of larger (smaller) variations in space are with faster (slower) motions in time. Therefore, to track spatially subtle distribution under feedback control with pointed actuation will always be accompanied by temporally abrupt transience. Inasmuch as distributed sensors and actuators are getting well-developed nowadays [8-12], 
distributed control is capable of remedying this situation. The small-gain theorem of Class $\mathrm{C} 4$ thus comes to play for robust syntheses of distributed control, on condition that the feedback controller is also belonging to the defined Sturm-Liouville dynamics.

In this paper, a general class of Sturm-Liouville dynamics is defined with Spatiotemporal Transfer Function (STF) obtained though the composite of modal decomposition and Laplace transform [5-7, 13-15]. The values of STF along imaginary axis thus define mode-frequency responses, whereon a $2 \mathrm{D}-H_{\infty}$ norm is created in mode-frequency domain that is employed to prove the small-gain theorem in Class C4. This newly developed mode-frequency domain makes possible to loop-shape feasible Sturm-Liouville controllers to guarantee dissipativity, well-posedness, passivity and exponential stability of the resulting closed-loop. Without the small-gain theorem in Class $\mathrm{C} 4$, robust control of Sturm-Liouville plants can merely be based on the small-gain theorem in Class $\mathrm{C} 2$ or $\mathrm{C} 3$, which will results in less vigorous robustness or performance, which is discussed in more detail in the next paragraph.

There were candidate approaches to Sturm-Liouville dynamics control. Some are based on order-truncated plants, for examples [16-20], which can employ the small-gain theorem in Class C3. Thereof, the spatiotemporally robust performance is not guaranteed at the stage of finite-dimensional syntheses. Another two types are involved in modelling with infinite-order transfer-functions [21-24] and identification with fraction-order transfer-functions [25-29], respectively, which can apply the small-gain theorem in Class C2 toward robust performance with merely well-posedness and dissipativity. Besides, they were not developed originally for distributed sensing and actuation. The other is $\mathrm{nD}$ state-space robust control [30-35] extended from $1 \mathrm{D}$ robust state-space synthesis to $\mathrm{nD}$ version in space-time domain. It can merely employ the small-gain theorem in Class C2 to guarantee well-posedness and dissipativity. For these reasons, this work show how the small-gain theorem in Class C4 leads to modal-spectral loopshaping of Sturm-Liouville controllers to guarantee Hurwitz, dissipativity, passivity and well-posedness, which is beyond the capacities of the above approaches.

Including this introduction section, this paper is organized into seven sections. Section 2 defines through spatiotemporal transfer-functions the fraction-order class of Sturm-Liouville dynamics. Section 3 develops mode-frequency responses. Therein the $H_{\infty}$-norm is extended to be $2 \mathrm{D}-H_{\infty}$ norm in mode-frequency domain and geometrical isomorphism is created between space-time domain and mode-frequency domain. With such a $2 \mathrm{D}-H_{\infty}$ norm, Section 4 proves that Hurwitz, passivity and dissipativity are equivalent to one another for the defined class of Sturm-Liouville dynamics. On grounds of the above sections, Section 5 proves the small-gain theorem of Class C4. Section 6 applies this theorem into $\mu$-loopshaping in mode-frequency domain. Section 7 concludes the present work.

\section{Fraction-Order Sturm-Liouville Dynamics}

Consider the following two types of Sturm-Liouville operator A operated in bounded regions $\Omega$ :

Type I: $\mathrm{A} \phi=-(1 / \rho) \nabla \cdot(k \nabla \phi)$ in $\Omega$,

$$
\alpha \phi+\beta \nabla \phi \cdot \hat{n}=0 \quad \text { on } \partial \Omega
$$

Type II: $\mathrm{A} \phi=(1 / \rho) \nabla^{2}\left(k \nabla^{2} \phi\right)$ in $\Omega$,

$$
\alpha \phi+\beta \nabla \phi \cdot \hat{n}=0 \wedge \alpha k \nabla^{2} \phi+\beta \nabla\left(k \nabla^{2} \phi\right) \cdot \hat{n}=0 \text { on } \partial \Omega,
$$

where $\rho(x)>0, k(x)>0, \forall x \in \Omega$, and $\alpha(x) \cdot \beta(x) \geq 0$ for all $x \in \partial \Omega$. Here we are concerned with von-Neumann, Dirichlet and Robin types of boundary conditions. The spatial operator A above is positive-definite and self-adjoint under the inner-product:

$$
\langle\psi, \phi\rangle_{\Omega}=\int_{\Omega} \rho(x) \phi^{*}(x) \psi(x) d V,
$$

and its inverse is a compact operator in $L_{2}(\Omega)$. Thus, its eigenvalues are all positive, and its eigenfunctions can constitute an real, feasible, orthonormal, and complete basis of $L_{2}(\Omega)$. Here we denote by $\Lambda$ the countable set of A's eigenvalues and by $\left\{\phi_{\lambda}\right\}_{\lambda \in \Lambda}$ the set of corresponding eigenfunctions, i.e.

$$
\mathrm{A} \phi_{\lambda}=\lambda \phi_{\lambda} \text { in } \Omega, \alpha \phi_{\lambda}+\beta \nabla \phi_{\lambda} \cdot \hat{n}=0 \text { on } \partial \Omega \text {. }
$$

With respect to the eigenfunctions set $\left\{\phi_{\lambda}\right\}_{\lambda \in \Lambda}$, Galerkin transform $G$ from spatial functions to modal functions, $F(\lambda)=\mathrm{G}[f(x)]$, is defined by

$$
F(\lambda) \equiv \int_{\Omega} \rho(x) \phi_{\lambda}(x) f(x) d x .
$$

Completeness and orthonormality of $\left\{\phi_{\lambda}\right\}_{\lambda \in \Lambda}$ jointly imply that the Galerkin transform $G$ has a unique inverse $\mathrm{G}^{-1}$,

$$
\begin{gathered}
f(x)=\mathrm{G}^{-1}[F(\lambda)]: \\
f(x) \equiv \sum_{\lambda \in \Lambda} F(\lambda) \phi_{\lambda}(x) .
\end{gathered}
$$

Then, Laplace-Galerkin transform $\mathrm{H}$ from spatial-temporal functions to modal-complex functions is defined by the composite of Galerkin transform and Laplace transform:

$$
H=L G=G L \text {; }
$$

explicitly,

$$
F(\lambda, s) \equiv \mathrm{H}[f(x, t)]=\int_{0^{-}}^{\infty} \int_{\Omega} e^{-s t} \rho(x) \phi_{\lambda}(x) f(x, t) d x d t .
$$


Here the domain of one-side Laplace transform, denoted by $\Gamma$, is an infinite line parallel to the imaginary axis, whereon the integral in (4) is converged. Accordingly, the inverse of Laplace-Galerkin transform $\mathrm{H}^{-1}$ is the composite of the inverse of Laplace transform and that of Galerkin transform, that is,

$$
\mathrm{H}^{-1}=\mathrm{G}^{-1} \mathrm{~L}^{-1}=\mathrm{L}^{-1} \mathrm{G}^{-1}
$$

explicitly,

$$
f(x, t) \equiv \mathrm{H}^{-1}[F(\lambda, s)]=\frac{1}{2 \pi j} \sum_{\lambda \in \Lambda} \int_{\Gamma} F(\lambda, s) \phi_{\lambda}(x) e^{t s} d s .
$$

There are two basic properties about Galerkin and Laplace transforms:

$$
\mathrm{G}[\mathrm{A} f(x, t)]=\lambda \mathrm{G}[f(x, t)]
$$

based on Green's identity, and

$$
\mathrm{L}\left[\mathrm{D}_{t} f(x, t)\right]=s \mathrm{~L}[f(x, t)],
$$

where $D_{t}$ denotes the temporal differentiation. Following these two properties, the Laplace-Galerkin transform $\mathrm{H}$ is of

$$
\mathrm{H}\left[h\left(\mathrm{~A}, \mathrm{D}_{t}\right) f(x, t)\right]=h(\lambda, s) \cdot \mathrm{H}[f(x, t)],
$$

where $h$ is a ratio of two expressions of finite or some infinite length constructed from two independent variables, one standing for space and the other for time, allowing for the operations of addition, subtraction, multiplication, integer exponents in time, and fraction-order exponents in space. For example,

$$
\mathrm{H}\left[\frac{\mathrm{D}_{t}-\mathrm{A}^{1 / 2}}{\mathrm{D}_{t}+\mathrm{A}^{1 / 2}} f(x, t)\right]=\frac{s-\sqrt{\lambda}}{s+\sqrt{\lambda}} F(\lambda, s) .
$$

This work considers a general Sturm-Liouville dynamics $\hat{P}$ including the feedback controller that is to be synthesized:

$$
\begin{gathered}
M\left(\mathrm{~A}, \mathrm{D}_{t}\right) \psi=N\left(\mathrm{~A}, \mathrm{D}_{t}\right) q \text { in } \Omega \times[0, \infty), \\
\mathrm{B}\left(\mathrm{A}^{j-1} \psi\right)=0, j=1,2, \cdots \text { on } \Omega \times[0, \infty),
\end{gathered}
$$

where the operator B indicates the Robin boundary condition defined in Type I and Type II Sturm-Liouville operators. Therein $M$ and $N$ are of temporal integral-order and allow for spatial fraction-order of $\mathrm{A}$, but the spatiotemporal orders of $N$ is not larger than that of $M$. Performing Laplace-Galerkin transform $\mathrm{H}$ on the Sturm-Liouville dynamics in (8)-(9) yields its spatiotemporal transfer function:

$$
P(\lambda, s)=\frac{N(\lambda, s)}{M(\lambda, s)}
$$

For any proper dynamics $\hat{P}$ as described in (10), it is defined that the set $\{s: M(\lambda, s)=0, \forall \lambda \in \Lambda\}$ contains all poles of $P$, and similarly to zeros. Note that unstable poles-zeros cancellation is not considered in this work. Besides, the impulse response of $\hat{P}$ is defined by $p(x, t)=\mathrm{H}^{-1}[P(\lambda, s)]$.

\section{Geometrical Isomorphism}

Define the Fourier-Galerkin transform $R$ by the composite of Galerkin transform and Fourier transform, that is, $R=F G=G F$; explicitly,

$$
\begin{aligned}
& \hat{F}(\lambda, \omega) \equiv \mathrm{R}[f(x, t)] \\
& =\int_{-\infty}^{\infty} \int_{\Omega} \rho(x) \varphi^{*}(\lambda, \omega, x, t) f(x, t) d x d t .
\end{aligned}
$$

where the kernel $\phi: \Lambda \times \mathfrak{R} \times \Omega \times \mathfrak{R} \rightarrow \mathrm{C}$ is

$$
\varphi(\lambda, \omega, x, t) \equiv \frac{e^{j \omega t}}{\sqrt{2 \pi}} \phi_{\lambda}(x)
$$

Accordingly, the inverse of Fourier-Galerkin transform $\mathrm{R}^{-1}=\mathrm{G}^{-1} \mathrm{~F}^{-1}=\mathrm{F}^{-1} \mathrm{G}^{-1}$ is explicitly

$$
f(x, t) \equiv \mathrm{R}^{-1}[\hat{F}(\lambda, \omega)]=\sum_{\lambda \in \Lambda} \int_{-\infty}^{\infty} \varphi(\lambda, \omega, x, t) \hat{F}(\lambda, \omega) d \omega
$$

Fourier-Galerkin transform for one-side temporally spatial functions can be calculated by Laplace-Galerkin transform in (4)-(5) with the infinite line $\Gamma$ being the imaginary axis, provided that the underlying integral in (11) is converged. Consider a proper dynamics $\hat{P}$ with the spatiotemporal transfer function $P(\lambda, s)$, and denote its input by $f$ and output by $y$, i.e. $P(\lambda, s)=Y(\lambda, s) / F(\lambda, s)$. Then,

$$
\hat{Y}(\lambda, \omega)=P(\lambda, j \omega) \cdot \hat{F}(\lambda, \omega),
$$

where

$$
\begin{aligned}
& \hat{Y}(\lambda, \omega)=\mathrm{R}[y(x, t)]=1 / \sqrt{2 \pi} Y(\lambda, j \omega), \\
& \hat{F}(\lambda, \omega)=\mathrm{R}[f(x, t)]=1 / \sqrt{2 \pi} F(\lambda, j \omega),
\end{aligned}
$$

and $P(\lambda, j \omega)$ defines the mode-frequency response of the dynamics $\hat{P}$.

Since both Fourier transform and Galerkin transform are geometric isomorphic, i.e. one to one, linear and inner-product conserved, the pair of Fourier-Galerkin transforms $\left(\mathrm{R}, \mathrm{R}^{-1}\right)$ is of geometric isomorphism between space-time domain and mode-frequency domain. Specifically it implies 2-norm conservation, that is,

$$
\int_{0}^{\infty} \int_{\Omega} \rho(x)|f(x, t)|^{2} d x d t=\sum_{\lambda \in \Lambda} \int_{-\infty}^{\infty}|\hat{F}(\lambda, \omega)|^{2} d \omega,
$$


or succinctly written by $\|f\|=\|\hat{F}\|$. Further to define the 2D- $H_{\infty}$ norm of $P$ by

$$
\|P\|_{\infty} \equiv \max _{\lambda \in \Lambda, \omega \in \mathfrak{R}^{+}}|P(\lambda, j \omega)|
$$

Then, based on the 2-norm conservation in (15), the $L_{2}$-gain of the dynamics $\hat{P}$ equals the $2 \mathrm{D}-H_{\infty}$ norm of $P$, since

$$
\|\hat{P}\| \equiv \sup _{f} \frac{\|y\|}{\|f\|}=\sup _{\hat{F}} \frac{\|\hat{Y}\|}{\|\hat{F}\|} \leq \max _{\lambda \in \Lambda, \omega \in \Re^{+}}|P(\lambda, j \omega)|=\|P\|_{\infty},
$$

where the equality holds when $|\hat{F}|$ is a Dirac distribution in the mode-frequency domain at the argument of the maximum. With (16), $\|P\|_{\infty}<\gamma$ corresponds to the following $L_{2}$-gain performance in space-time domain:

$$
\int_{0}^{T} \int_{\Omega} \rho(x)|y(x, t)|^{2} d x d t<\gamma^{2} \int_{0}^{T} \int_{\Omega} \rho(x)|f(x, t)|^{2} d x d t, \forall T \in[0, \infty) .
$$

\section{Spatiotemporal Stability}

The legitimacy of $2 \mathrm{D}-H_{\infty}$ norm as a metric of robustness is justified by the proof that bounded 2D- $H_{\infty}$ norm implies Hurwitz, passivity, and dissipativity.

Definition 1: Hurwitz of a proper dynamics $\hat{P}$ is defined by that all of its poles are in the left-half plane, which implies exponential decay. Passivity is defined by that its impulse response $p: \Omega \times \mathfrak{R}^{+} \rightarrow \mathfrak{R}$ has $\lim _{t \rightarrow \infty} p(x, t)=0$ for almost everywhere $x \in \Omega$ in the sense of $L_{2}(\Omega)$. Dissipativity is defined by bounded $L_{2}$-gain, i.e.

$$
\sup _{\|f\|_{\Omega \times \Re^{+}} \| \hat{P} f}\|\|_{\Omega \times \Re^{+}}<\infty . \#
$$

It follows to prove that Hurwitz, passivity, and dissipativity are equivalent to one another.

Lemma 1: Hurwitz and passivity are equivalent to each other.

Proof. For a proper dynamics $\hat{P}$, its impulse response $p$ is known as

$$
p(x, t)=\mathrm{G}^{-1} \mathrm{~L}^{-1}[P(\lambda, s)] \equiv \mathrm{G}^{-1}\left[p_{\lambda}(t)\right]=\sum_{\lambda \in \Lambda} \phi_{\lambda}(x) p_{\lambda}(t)
$$

By Definition 1, if $\hat{P}$ is not Hurwitz, then there exists at least one pole on the imaginary axis or in the right-half plane. This implies that some $\lambda$-mode response $p_{\lambda}(t)$ will not converge to zero as the time $t \rightarrow \infty$. Since $\left\{\phi_{\lambda}\right\}_{\lambda \in \Lambda}$ is a linearly independent set because of being orthogonal to one another, the impulse response $p(x, t)$ will not converge to zero for all $x \in \Omega$ as $t \rightarrow \infty$. That is, passivity implies
Hurwitz.

Since $\left\{\phi_{\lambda}\right\}_{\lambda \in \Lambda}$ is an orthonormal, complete basis of $L_{2}(\Omega),(18)$ implies

$$
\|p\|_{\Omega}^{2}(t)=\sum_{\lambda \in \Lambda}\left|p_{\lambda}(t)\right|^{2}
$$

for any time $t$. If the dynamics $\hat{P}$ is Hurwitz, then there exist bounded values $c_{\lambda}>0, a_{\lambda}<0, t_{\lambda} \geq 0$ such that $\left\|p_{\lambda}(t)\right\|^{2} \leq c_{\lambda} e^{a_{\lambda} t}$ as $t \geq t_{\lambda}$ for all $\lambda \in \Lambda$, which is known from 1D stability. This furthers to imply

$$
\|p\|_{\Omega}^{2}(t) \leq c_{0} \sum_{\lambda \in \Lambda} e^{a_{\lambda} t} \text { for } \forall t \geq t_{0},
$$

where $t_{0} \equiv \max _{\lambda \in \Lambda} t_{\lambda}$ and $c_{0} \equiv \max _{\lambda \in \Lambda} c_{\lambda}$. The set $\left\{a_{\lambda}: \lambda \in \Lambda\right\}$ can be chosen to have distinct members, therefore there exists $\varepsilon>0$ small enough such that

$$
\|p\|_{\Omega}^{2}(t) \leq c_{0}\left(e^{a_{0} t}+e^{\left(a_{0}-\varepsilon\right) t}+e^{\left(a_{0}-2 \varepsilon\right) t}+\cdots\right) \text { for } \forall t \geq t_{0},
$$

where $a_{0} \equiv \max _{\lambda \in \Lambda} a_{\lambda}$. The above equation implies

$$
\|p\|_{\Omega}^{2}(t) \leq c_{0} \frac{e^{a_{0} t}}{1-e^{-\varepsilon t}} \text { for } \forall t \geq t_{0}
$$

thereby $\|p\|_{\Omega}^{2}(t) \rightarrow 0$ as $t \rightarrow \infty$. That is, $\lim _{t \rightarrow \infty} p(x, t)=0$ for almost everywhere $x \in \Omega$ in the sense of $L_{2}(\Omega)$, so Hurwitz implies passivity. \#

Lemma 2: Hurwitz and dissipativity are equivalent to each other.

Proof. As the signal $f(x, t)=\phi_{\lambda}(x) v(t)$ with a specific $\lambda \in \Lambda$ is input to the dynamics $\hat{P}$, it will output

$$
y(x, t)=\phi_{\lambda}(x) \int_{0^{-}}^{t} p_{\lambda}(t-\tau) v(\tau) d \tau \equiv \phi_{\lambda}(x) y_{\lambda}(t) .
$$

Suppose some roots $s$ of $M(\lambda, s)=0$ are on imaginary axis or in right-half plane, then $p_{\lambda}$ is not exponentially bounded. Known from 1D convolution, $y_{\lambda}$ will be unbounded in the sense of $L_{2}\left(\Re^{+}\right)$as $v$ is bounded by $\|v\|_{\mathcal{R}^{+}}=1$. Since $\|y\|_{\Omega \times \mathcal{R}^{+}}=\left\|y_{\lambda}\right\|_{\mathcal{R}^{+}}$in such a case, this furthers to imply that $y$ is $L_{2}\left(\Omega \times \Re^{+}\right)$-unbounded as $\|f\|_{\Omega \times \Re^{+}}=\|v\|_{\mathfrak{R}^{+}}=1$. Therefore, the dynamics $\hat{P}$ is not dissipative if it is not Hurwitz, that is, dissipativity implies Hurwitz. As of such, for any non-Hurwitz Sturm-Liouville dynamics, its $2 \mathrm{D}-H_{\infty}$ can be defined to be unbounded.

Suppose the dynamics $\hat{P}$ to be Hurwitz, that is, all poles of $P$ are in the left-half plane. This implies that its $2 \mathrm{D}-H_{\infty}$ norm $\|P\|_{\infty} \equiv \max _{\lambda \in \Lambda, \omega \in \mathcal{R}^{+}}|P(\lambda, j \omega)|$ is bounded, since there is no singularities $(\lambda, \omega) \in \Omega \times \Re^{+}$in the denominator $M(\lambda, j \omega)$. 
Since the $L_{2}$-gain of $\hat{P}$ equals the 2D- $H_{\infty}$ norm of $P$, as shown in (16), the dynamics $P$ is dissipative. That is, Hurwitz implies dissipativity. \#

Based on Lemma 1 and Lemma 2, we conclude the above discussion with the following theorem:

Theorem 1: For a proper Sturm-Liouville dynamics, Hurwitz, passivity, dissipativity, and bounded 2D- $H_{\infty}$-norm are equivalent to one another. \#

For a finite-dimensional, matrix-valued dynamics $\hat{P}$ with all entries being proper Sturm-Liouville dynamics, the 2D- $H_{\infty}$ norm of $P$ is defined by

$$
\|P\|_{\infty} \equiv \max _{\lambda \in \Lambda, \omega \in \Re^{+}} \bar{\sigma}(P(\lambda, j \omega)),
$$

where $\bar{\sigma}$ denotes the singular value of the underlying matrix. As of such, the $L_{2}$-gain of $\hat{P}$ is equal to the 2D- $H_{\infty}$ norm of $P$, since

$$
\|P\| \equiv \sup _{f} \frac{\|y\|_{\Omega \propto \mathcal{R}^{+}}}{\|f\|_{\Omega \propto \mathcal{R}^{+}}}=\sup _{\hat{F}} \frac{\|\hat{Y}\|_{\Lambda \propto \Re}}{\|\hat{F}\|_{\Lambda \curvearrowright \Re}}=\max _{\lambda \in \Lambda, 0 \in \mathcal{R}^{+}} \bar{\sigma}(P(\lambda, j \omega)) \equiv\|P\|_{\infty} .
$$

Definition 2: Any finite-dimensionally matrix-valued, proper Sturm-Liouville dynamics is defined to be spatiotemporally stable if all of its entries are Hurwitz, passive or dissipative. \#

Due to Pythagorean Proposition, a Sturm-Liouville dynamics matrix has bounded $L_{2}$-gain if and only if all of its entries are dissipative. Therefore, Theorem 1 still holds as Sturm-Liouville dynamics expands on being finite-dimensionally matrix-valued.

\section{Small Gain Theorem}

Suppose a nominal dynamics $M$ is feedback-connected by an unstructured uncertainty $\Delta$ bounded by $2 \mathrm{D}-H_{\infty}$ norm, $\|\Delta\|_{\infty} \leq \gamma^{-1}$, as shown in Figure 1, then the closed-loop dynamics is guaranteed to be well-posed and spatiotemporally stable if and only if $\|M\|_{\infty}<\gamma$. This is an equivalent statement of the small-gain theorem of Class $\mathrm{C} 4$, and proved here.

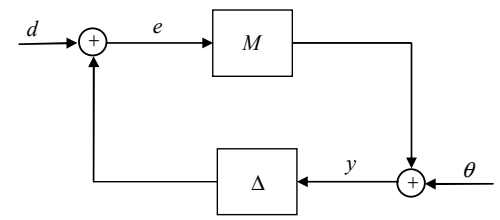

Figure 1. For Spatiotemporal Small Gain theorem

Theorem 2: Consider the closed-loop dynamics feedback-connected by any two proper Sturm-Liouville dynamics $\hat{M}$ and $\hat{\Delta}$ with compatible matrix dimensions. If $\|M\|_{\infty}<\gamma$ and $\|\Delta\|_{\infty} \leq \gamma^{-1}$, the closed loop must be of well-posedness and spatiotemporal stability.
Proof. Define the loop gain $A$ by $A=M \Delta$ or $A=\Delta M$. Firstly, we prove that if $\|A\|_{\infty}<1$, then firstly the inverse of $I-A$ is uniquely existent and has bounded $2 \mathrm{D}-H_{\infty}$ norm.

For any function $f \in L_{2}(\Lambda \times \Re)$, the sequence $\left(\left(I+A+\cdots+A^{n}\right) f\right)_{n=1}^{\infty}$ is Cauchy in $L_{2}(\Lambda \times \Re)$, for if $m>n$,

$$
\begin{aligned}
& \left\|\left(I+A+\cdots+A^{m}\right) f-\left(I+A+\cdots+A^{n}\right) f\right\|_{2} \\
= & \left\|A^{n+1} f+\cdots+A^{m} f\right\|_{2} \leq\left\|A^{n+1}\right\|_{\infty}\|f\|_{2}+\cdots+\left\|A^{m}\right\|_{\infty}\|f\|_{2} \\
\leq & \|f\|_{2}\left\{\|A\|_{\infty}^{n+1}+\cdots+\|A\|_{\infty}^{m}\right\} \leq\|f\|_{2} \sum_{r=n+1}^{\infty}\|A\|_{\infty}^{r} \\
= & \|f\|_{2} \frac{\|A\|_{\infty}^{n+1}}{1-\|A\|_{\infty}} .
\end{aligned}
$$

Since $\|A\|_{\infty}^{n+1} \rightarrow 0$ as $n \rightarrow \infty$, the left-hand side tends to zero as $m, n \rightarrow \infty$ with $m>n$. Since $L_{2}(\Lambda \times \Re)$ is a Banach space, the Cauchy sequence $\left(\left(I+A+\cdots+A^{n}\right) f\right)_{n}$ converges to a limit point $T f \in L_{2}(\Lambda \times \Re)$. On letting $m \rightarrow \infty$ in the above inequality we find

$$
\left\|T f-\left(I+A+\cdots+A^{n}\right) f\right\|_{2} \leq\|f\|_{2} \frac{\|A\|_{\infty}^{n+1}}{1-\|A\|_{\infty}} .
$$

This shows that $\left\|T-\left(I+A+\cdots+A^{n}\right)\right\|_{\infty}$ is bounded, and hence that $\|T\|_{\infty}$ is. Furthermore,

$$
\left\|T-\left(I+A+\cdots+A^{n}\right)\right\|_{\infty} \leq \frac{\|A\|_{\infty}^{n+1}}{1-\|A\|_{\infty}},
$$

which implies that

$$
I+A+\cdots+A^{n} \rightarrow T \text { as } n \rightarrow \infty .
$$

It remains to show that $T=(I-A)^{-1}$. For any $f \in L_{2}(\Lambda \times \Re)$, bounded 2D- $H_{\infty}$ norm of $I-A$ gives

$$
\begin{aligned}
(I-A) T f & =(I-A) \lim _{n \rightarrow \infty}\left(I+A+\cdots+A^{n}\right) f \\
& =\lim _{n \rightarrow \infty}(I-A)\left(I+A+\cdots+A^{n}\right) f \\
& =\lim _{n \rightarrow \infty} f-A^{n+1} f .
\end{aligned}
$$

We have

$$
\left\|A^{n+1} f\right\|_{2} \leq\left\|A^{n+1}\right\|_{\infty}\|f\|_{2} \leq\|A\|_{\infty}^{n+1}\|f\|_{2},
$$

and thus $(I-A) T f=f$, since $A^{n+1} f \rightarrow 0$ as $n \rightarrow \infty$. Similarly, $\quad T(I-A) f=f$, and then $T=(I-A)^{-1}$, 
measured by the $2 \mathrm{D}-H_{\infty}$ norm.

Secondly, the internal behavior of the closed-loop of Figure 1 is governed by

$$
\left[\begin{array}{l}
e \\
y
\end{array}\right]=\left[\begin{array}{cc}
(I-\Delta M)^{-1} & \Delta(I-M \Delta)^{-1} \\
M(I-\Delta M)^{-1} & (I-M \Delta)^{-1}
\end{array}\right]\left[\begin{array}{l}
d \\
\theta
\end{array}\right],
$$

On grounds of the above deduction, $\|\Delta M\|_{\infty} \leq\|\Delta\|_{\infty} \cdot\|M\|_{\infty}<1 \quad$ implies that $\quad(I-\Delta M)^{-1}$ is uniquely existent and 2D- $H_{\infty}$ norm -bounded, and similarly to $(I-M \Delta)^{-1}$. Therefore, all entries of the $2 \times 2$ block matrix in (21) are uniquely existent and $2 \mathrm{D}-H_{\infty}$ norm -bounded. Further to note that the feedback connection of two Sturm-Liouville dynamics is also belonging to Sturm-Liouville dynamics. Based on Theorem 1, the closed-loop dynamics is of well-posedness and spatiotemporally stable. Moreover, there is no any structure constraint on the uncertainty $\Delta$, so $\|\Delta M\|_{\infty}<1$ or $\|M \Delta\|_{\infty}<1$ for all $\|\Delta\|_{\infty} \leq \gamma^{-1}$ implies $\|M\|_{\infty}<\gamma$.\#

\section{Application to Loop Shaping}

Accommodating some extent of model uncertainties, the mode-frequency responses can be curve-fitted in the mode-Bode plot as a dense set:

$$
\left\{G_{0}\left(1+W_{1} \Delta_{1}\right):\left\|\Delta_{1}\right\|_{\infty} \leq 1\right\}
$$

where $G_{0}$ stands for a nominal plant. Therein the set $\left\{W_{1} \Delta_{1}:\left\|\Delta_{1}\right\|_{\infty} \leq 1\right\}$ contains model uncertainties, wherein the robustness weighting $W_{1}$ envelops all multiplicative perturbations $W_{1} \Delta_{1}$ in the modal-Bode magnitude plot. As shown in Figure 2a, the Sturm-Liouville controller $K$, preor post- composite to the plant, together with a negative unit feedback -1 executes the feedback compensation. The controller $K$ is to guarantee specified performance for all uncertain plants under consideration, i.e. robust performance. Here the performance is specified by $L_{2}$-gain as

$$
\begin{aligned}
& \int_{0}^{T} \int_{\Omega} \rho(x)\left|\hat{z}_{2}(x, t)\right|^{2} d x d t<\gamma^{2} \int_{0}^{T} \int_{\Omega} \rho(x)\left|\hat{w}_{2}(x, t)\right|^{2} d x d t \\
& \forall T \in[0, \infty)
\end{aligned}
$$

where the exogenous disturbances $w_{2}$ comprises the slow-time reference command and sensor noises of higher frequencies. Therein, the tracking error $e$ has been weighted by the performance weighting $W_{2}$, i.e. $z_{2}=W_{2} e$.

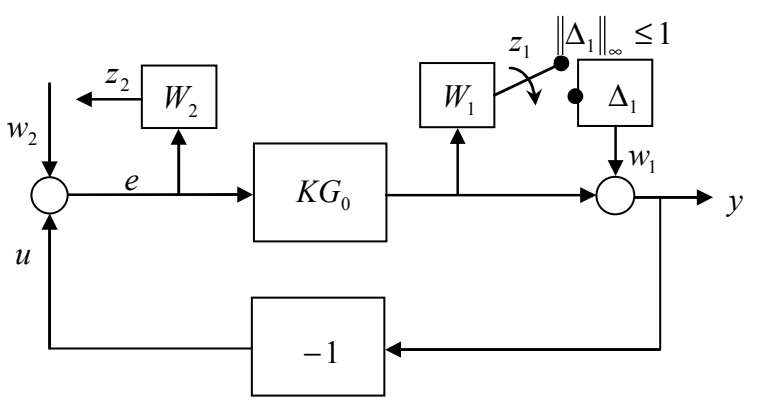

Figure 2a. Construction of generalized plant

Tracing the path of signal flow in Figure $2 \mathrm{a}$, one can transform the interconnection of blocks in Figure 2a into the feedback-interconnection of three blocks:

Generalized plant:

$$
P:\left[\begin{array}{c}
z_{1} \\
z_{2} \\
y
\end{array}\right]=\left[\begin{array}{ccc}
0 & W_{1} K G_{0} & W_{1} K G_{0} \\
0 & W_{2} & W_{2} \\
1 & K G_{0} & K G_{0}
\end{array}\right]\left[\begin{array}{c}
w_{1} \\
w_{2} \\
u
\end{array}\right] ;
$$

External feedback:

$$
u=-y
$$

Internal feedback 1:

$$
w_{1}=\Delta_{1} z_{1},\left\|\Delta_{1}\right\|_{\infty} \leq 1 .
$$

Therein the internal disturbance $w_{1}$ and stability variable $z_{1}$ are induced from modelling uncertainty $\Delta_{1}$. Moreover, if we replace the $L_{2}$-gain performance in (22) by such an internal feedback as

Internal feedback 2:

$$
w_{2}=\Delta_{2} z_{2},\left\|\Delta_{2}\right\|_{\infty} \leq 1
$$

then the robust performance of the original setting in Figure $2 \mathrm{a}$ is equivalent to the robust stability of the closed-loop system in Figure $2 b$. This equivalency is inferred from the small-gain theorem of Theorem 2. Substituting (24) into (23) yields the lower fractional transformation of Figure $2 b$, which is shown in Figure 2c. It becomes the feedback-interconnection of two blocks:

$$
\begin{aligned}
& \mathrm{M}=\left[\begin{array}{ll}
-\mathrm{W}_{1} \mathrm{~T} & \mathrm{~W}_{1} \mathrm{~T} \\
-\mathrm{W}_{2} \mathrm{~S} & \mathrm{~W}_{2} \mathrm{~S}
\end{array}\right] \text { and } \Delta=\left[\begin{array}{cc}
\Delta_{1} & 0 \\
0 & \Delta_{2}
\end{array}\right] ; \\
& \mathrm{z}=\left[\begin{array}{l}
\mathrm{z}_{1} \\
\mathrm{z}_{2}
\end{array}\right], \quad \mathrm{w}=\left[\begin{array}{l}
\mathrm{w}_{1} \\
\mathrm{w}_{2}
\end{array}\right]
\end{aligned}
$$

where $S$ is the sensitivity function $1 /\left(1+K G_{0}\right)$, and $T$ is its complementary function, i.e. $S+T=1$. The first requirement of the controller $K$ is to make the sensitivity function $S$ spatiotemporally stable.

If the $\Delta$-structured singular value $\mu_{\Delta}$ is defined by 


$$
\mu_{\Delta}(M)=\frac{1}{\min \{\bar{\sigma}(\Delta): \operatorname{det}(I-M \Delta)=0\}},
$$

then based on Theorem 2 assisted with Theorem 1 and Nyquist criterion, the spatiotemporally robust stability of Figure 2c is guaranteed, so is spatiotemporally robust performance of Figure 2a, if and only if

$$
\mu_{\Delta}(M(\lambda, j \omega)) \leq 1 \text {, for all }(\lambda, \omega) \in \Lambda \times \Re^{+} .
$$

Substituting (27) into (28) yields

$$
\mu_{\Delta}(M)=\frac{1}{\min \left\{\max \left(\left|\Delta_{1}\right|,\left|\Delta_{2}\right|\right): 1+W_{1} T \Delta_{1}-W_{2} S \Delta_{2}=0\right\}},
$$

which, after careful calculation, is explicitly to be

$$
\mu_{\Delta}(M)=\left|W_{1} T\right|+\left|W_{2} S\right|
$$

Therefore, the set of sensitivity functions with spatiotemporally robust performance in the original setting of Figure $2 \mathrm{a}$ is

$$
\left\{\begin{array}{l}
S:\left|W_{1}(\lambda, j \omega)(1-S(\lambda, j \omega))\right| \\
+\left|W_{2}(\lambda, j \omega) S(\lambda, j \omega)\right|<1, \forall(\lambda, \omega) \in \Lambda \times \Re^{+}
\end{array}\right\}
$$

Finally, we know that any feasible loop $S$ is a spatiotemporally stable transfer function and a member of (30).

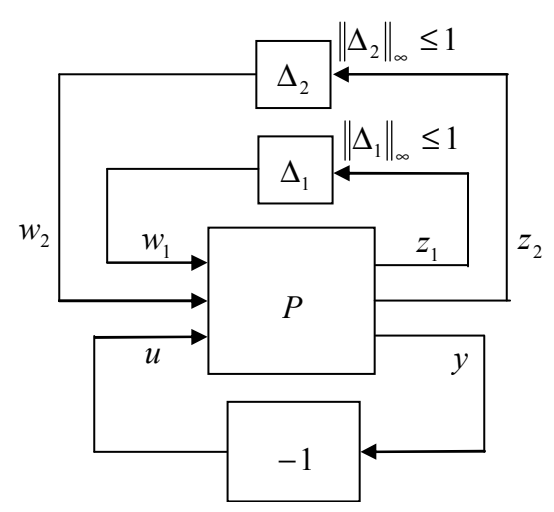

Figure 2b. Robust stability of Figure $2 a$

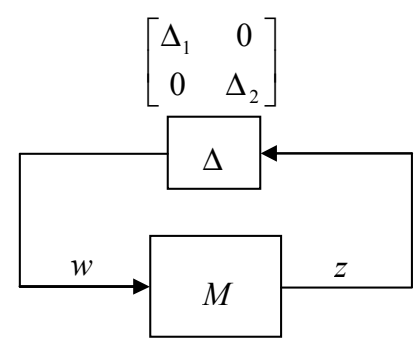

Figure 2c. Fractional transformation of Figure $2 b$

A graphical interpretation of robust performance is shown in Figure 3, where the line segment of $S+T=1$ inside the diamond of $\mu_{\Delta}(M)=1$ represents the set of all feasible loops $S$. In figure 3, we also demonstrate the conservatism of singular value $\bar{\sigma}(M)$ and the risk of spectral radius $\rho(M)$ as metrics of robust performance.

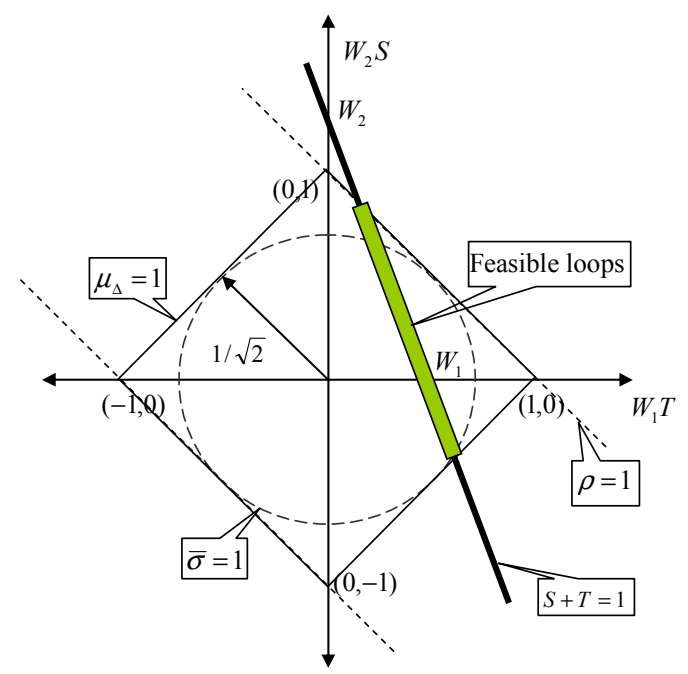

Figure 3. Graphical interpretation of robust performance

\section{Conclusion}

Small Gain theorem is always regarded to the most fundamental as to Robust Control. This paper implants an elegant small-gain theorem to Sturm-Liouville systems control, the closed-loop of which is guaranteed of well-posedness, exponential decay, passivity and dissipativity. To approach this, it compromises:

1. The definition of fraction-order Sturm-Liouville dynamics through spatiotemporal transfer-functions;

2. The creation of geometrical isomorphism and 2D- $H_{\infty}$ norm in the mode-frequency domain;

3. The proofs of the equivalency of Hurwitz, passivity, dissipativity to bounded 2D- $H_{\infty}$ norm, and the Sturm-Liouville Small Gain theorem; and

4. The derivation of $\mu$-loopshaping in the mode-frequency domain as an application example of Sturm-Liouville Small Gain theorem.

\section{Acknowledgements}

Thanks for the financial support from Taiwan under NSC 103-2221-E-194-041.

\section{References}

[1] H. K. Khalil, Nonlinear Systems Second Edition, Prentice-Hall Inc., Upper Saddle River, New Jersey, 1996.

[2] A. W. Naylor and G. R. Sell, Linear Operator Theory in Engineering and Science, Springer-Verlag New York Inc., New York, 1982.

[3] J. Doyle, B. Francis, A. Tannenbaum, Feedback Control Theory, Macmillan, New York, 1992. 
[4] K. Zhou, J. C. Doyle and K. Glover, Robust and Optimal Control, Prentice-Hall Inc., Upper Saddle River, New Jersey, 1996.

[5] B.-S. Hong, P.-J. Su, C.-Y. Chou, C.-I. Hung, Realization of non-Fourier phenomena in heat transfer with $2 \mathrm{D}$ transfer function, Appl. Math. Model. 35 (8) (2011) 4031-4043.

[6] C.-Y. Chou, System Identification and Feedback Control of Non-Fourier Heat Transfer with 2D Transfer Function, Doctoral Dissertation, National Chung Cheng University, Taiwan, 2012.

[7] B.-S. Hong, C.-Y. Chou, T.-Y. Lin, 2D transfer function modeling of thermoacoustic vibration engines with boundary heat-flux control, Asian J. Control (to appear).

[8] A. Preumont, A. François, P. De Man, N. Loix, K. Henrioulle, Distributed sensors with piezoelectric films in design of spatial filters for structural control, J. Sound Vibr. 282 (3-5) (2005) 701-712.

[9] A.W. Brown, B.G. Colpitts, K. Brown, Distributed sensor based on dark-pulse Brillouin scattering, IEEE Photon. Technol. Lett. 17 (7) (2005) 1501-1503.

[10] T.V. karnaukhova, E.V. Pyatetskaya, Basic relations of the theory of thermoviscoelastic plates with distributed sensors, Int. Appl. Mech. 45 (6) (2009) 660-669.

[11] T.V. Karnaukhova, E.V. Pyatetskaya, Basic equations for termoviscoelastic plates with distributed actuators under monoharmonic loading, Int. Appl. Mech. 45 (2) (2009) 200-214.

[12] C. Menon, F. Carpi, D.D. Rossi, Concept design of novel bio-inspired distributed actuators for space applications, Acta Astronaut. 65 (5-6) (2009) 825-833.

[13] R. Rabenstein, L. Trautmann, Multidimensional transfer function models, IEEE Trans. Circuits Syst. Fund. Theor. Appl. 49 (6) (2002) 852-861.

[14] R. Rabenstein, L. Trautmann, Digital sound synthesis of string instruments with the functional transformation method, Signal Process. 83 (8) (2003) 1673-1688.

[15] B.-S. Hong, Construction of 2D isomorphism for 2D $H_{1}$ -control of Sturm-Liouville Systems, Asian J. Control 12 (2) (2010) 187-199.

[16] B. Bamieh, F. Paganini, M.A. Dahleh, Distributed control of spatially invariant systems, IEEE Trans. Autom. Control 47 (7) (2002) 1091-1107.

[17] D.M. Gorinevsky, G. Stein, Structured uncertainty analysis of robust stability for multidimensional array systems, IEEE Trans. Autom. Control 48 (9) (2003) 1557-1568.

[18] G.E. Stewart, D.M. Gorinevsky, G.A. Dumont, Two-dimensional loop shaping, Automatica 39 (5) (2003) 779-792.

[19] G.E Stewart, D.M. Gorinevsky, G.A Dumont, Feedback controller design for a spatially distributed system: the paper machine problem, IEEE Trans. Control. Syst. Technol. 11 (5) (2003) 612-628.

[20] D.M. Gorinevsky, S. Boyd, G. Stein, Design of low-bandwidth spatially distributed feedback, IEEE Trans. Autom. Control 53 (1) (2008) 257 - 272.

[21] F.M. Callier, C.A. Desoer, An algebra of transfer functions for distributed linear time-invariant systems, IEEE Trans. Circuits Syst. 25 (9) (1978) 651-662.

[22] F.M. Callier, J. Winkin, LQ-optimal control of infinite-dimensional systems by spectral factorization, Automatica 28 (4) (1992) 757-770.

[23] F.M. Callier, L. Dumortier, J. Winkin, On the nonnegative self-adjoint solutions of the operator Riccati equation for infinite dimensional systems, Integr. Equ. Oper. Theory 22 (2) (1995) 162-195.

[24] P. Grabowski, F.M. Callier, Boundary control systems in factor form: transfer functions and input-output maps, Integr. Equ. Oper. Theory 41 (1) (2001) 1-37.

[25] I. Podlubny, Fractional-order systems and $P I^{\lambda} D^{\mu}$ -controllers, IEEE Trans. Autom. Control 44 (1) (1999) 208-214.

[26] D. Valerio, J.S. da Costa, Tuning of fractional Controllers Minimising $\mathrm{H}_{2}$ and $\mathrm{H}_{1}$ Norms, Acta Polytech. Hung. 3 (4) $200655-70$.

[27] B.M. Vinagre, V. Feliu, Optimal fractional controllers for rational order system: a special case of the Wiener-Hopf spectral factorization method, IEEE Trans. Autom. Control 52 (12) (2007) 2385-2389.

[28] C.A. Monje, B.M. Vinagre, V. Feliu, Y.-Q. Chen, Tuning and auto-tuning of fractional order controllers for industry applications, Control Eng. Practice 16 (2008) 798-812.

[29] F. Padula, A. Visioli, Tuning rules for optimal PID and fractional-order PID controllers, J. Process Control 21 (2011) 69-81.

[30] M.E. Valcher, On the internal stability and asymptotic behavior of 2-D positive systems, IEEE Trans. Circuits Syst. 44 (7) (1997) 602-613.

[31] M.E. Valcher, State-space descriptions and observability properties of spatiotemporal finite-dimensional autonomous behaviors, Syst. Control Lett. 44 (2) (2001) 91-102.

[32] E. Fornasini, M.E. Valcher, Controllability and reachability of 2-D positive system: A Graph Theoretic Approach, IEEE Trans. Circuits Syst. 52 (3) (2005) 576-585.

[33] P. Lancaster, I. Zaballa, Diagonalizable quadratic eigenvalue problems, Mech. Syst. Signal Pr. 23 (4) (2009) 1134-1144.

[34] J. Carlos, Z. Anaya, Diagonalization of quadratic matrix polynomials, Syst. Control Lett. 59 (2) (2010) 105-113.

[35] Z. Shu, J. Lam, H. Gao, B. Du, L. Wu, Positive observers and dynamic output-feedback controllers for interval positive linear systems, IEEE T. Circuits.-I 55 (10) (2008) 3209-3222. 